\title{
Seven Autophagy-Related IncRNAs Associated With Clinical Prognosis in Hepatocellular Carcinoma.
}

\author{
Yaping Zhou \\ Shihezi University \\ Liu Yang \\ Shihezi University \\ Xiangxin Zhang \\ Shihezi University \\ Xiaotong Zhao \\ Shihezi University \\ Jianfeng Fu ( $D$ dxpjf@163.com) \\ General Hospital of Xinjiang Military Region, Xinjiang \\ Lu Wang \\ Bayingol Mongolian Autonomous Prefecture People's Hospital
}

\section{Research Article}

Keywords: Hepatocellular carcinoma, Autophagy-related IncRNAs, the Risk prediction model

Posted Date: January 4th, 2021

DOI: https://doi.org/10.21203/rs.3.rs-134718/v1

License: (1) This work is licensed under a Creative Commons Attribution 4.0 International License. Read Full License 


\section{Abstract}

Background: LncRNA may be involved in the occurrence, metastasis, and chemical reaction of hepatocellular carcinoma (HCC) through various pathways associated with autophagy. Therefore, it is urgent to reveal more autophagy-related IncRNAs, explore these IncRNAs' clinical significance, and find new targeted treatment strategies.

Methods: In our study, RNA-seq and clinical data of normal and HCC patients were obtained from the TCGA database, and autophagy genes were obtained from the human autophagy database.

Results: The risk prediction model containing seven autophagy-related IncRNAs was constructed. Overall survival (OS) curves show that the high-risk group patients significantly shorter than the low-risk group ( $\mathrm{P}=2.292 \mathrm{e}-10)$, and the five years survival rate of the high-risk group (HR 0.286, 95\% $\mathrm{Cl} 0.199-0.411$ ) is less than half of the low-risk group ( $\mathrm{HR} 0.694,95 \% \mathrm{Cl} 0.547-0.77$ ). Univariate Cox regression indicated that risk score of the risk prediction model $(P<0.001,95 \% \mathrm{Cl} 1.210-1.389), T(P<0.001,95 \% \mathrm{Cl} 1.443-2.287)$, and stage $(P<0.001$ , $95 \% \mathrm{Cl} 1.466-2.408$ ) were independent prognostic indicators. However, only the risk score remains the independent prognostic indicator $(\mathrm{P}<0.001,95 \% \mathrm{Cl} 1.197-1.400)$ based on the multivariate analysis. This risk model's prediction efficiency is significantly higher than other clinicopathological factors for 1-, 3- and 5-year survival rate prediction (AUC are $0.853,0.794$, and 0.764 , respectively). Remarkably, the 7 autophagy-related IncRNAs may participate in Spliceosome, Cell cycle, RNA transport, DNA replication, and mRNA surveillance pathway and be related to the biological process of RNA splicing and mRNA splicing.

Conclusion: In conclusion, the 7 autophagy-related IncRNAs might be promising prognostic and therapeutic targets for HCC.

\section{Introduction}

Hepatocellular carcinoma (HCC), as the most common type of liver cancer [1], with high cancer-related mortality and poor prognosis worldwide [2], makes a threat to public health. Alcohol, aflatoxin, and hepatitis can increase HCC risk, and genetic and epigenetic changes can promote malignancy[3.4]. At present, surgical resection remains the most common treatment option for patients with HCC. Because of tumor metastasis and relapse [5, 6], the prognosis is dissatisfying. Most patients with advanced HCC often have low 5-year survival [7]. Currently, molecular alterations of HCC have been reported in some studies[8], and those molecular mechanisms can be explored further in the diagnosis and treatment of HCC [9-11]. However, these efforts have not made a significant improvement in patient survival. Therefore, it is necessary to identify more potential biomarkers that may be related to HCC carcinogenesis.

As a highly selective quality control, mechanism autophagy is the key to maintaining homeostasis in physiological and pathological conditions [12], such as adapting to metabolic stress, removing dangerous cargo, and renovating during differentiation and development, genomic prevention damage [13]. However, more and more evidence indicated that hat autophagy could also help tumor occurrence, maintenance, and development [14]. In pancreatic cancer [15], autophagy is a metabolic requirement for cancer cells' immune evasion, allowing tumors to achieve optimal proliferation in vitro and vivo. Besides, autophagy is necessary for tumor cell migration and metastasis because its inhibition will block cell migrates and metastasis [16]. In HCC, autophagy can promote tumor cells' metastasis by upregulating the expression of MCT1 and the activation of the Wnt/ $\beta$-catenin signaling pathway [17]. The epithelialmesenchymal can also be activated by autophagy to promote cancer cell invasion [18]. Thus some researchers have tried to find new targeted treatment strategies for HCC by studying autophagy pathways.

Long non-coding RNAs (IncRNAs) are a class of RNA transcripts that consist of more than 200 nucleotides in length and exhibit limited protein-coding capacity [19]. However, With in-depth exploration, IncRNA has been found to perform essential functions in various biological processes such as post-transcriptional regulation, transcriptional regulation, and chromatin modification [20]. Some studies have also reported that IncRNAs regulate many aspects of cancer progression and affect different malignant behaviors, such as cancer cell proliferation, apoptosis, and metastasis [21, 22]. Remarkably, owing to the complexity and diversity, IncRNA's abnormal expression will promote the occurrence of a variety of tumors, such as cervical carcer[23], esophageal squamous cell carcinoma [24], and lung adenocarcinoma [25]. Autophagy has been considered to play a dual and contradictory role in carcinogenesis. The exact mechanisms that result in autophagy in cancer still need to be further explored[26]. IncRNA participates in the occurrence, invasion, metastasis, prognosis, and chemoresistance of HCC by regulating various pathways related to autophagy[27-29]. However, these studies only concentrated on single or a few IncRNAs for HCC. Therefore, we used the IncRNA expression profiles of the TCGA database to explore new biomarkers of autophagy-related IncRNAs that are only closely related to and predict patients' prognosis with HCC.

\section{Materials And Methods}




\subsection{Data obtaining and processing}

The entire sequencing profile data of patients with liver hepatocellular carcinoma (HCC) were obtained from The Cancer Genome Atlas (TCGA, https://cancergenome.nih.gov/) database. According to the gene annotations from the GENCODE project (https://www.gencodegenes.org/) [30], the IncRNA and protein-coding gene profile data were further classified. Besides, the patient's corresponding clinical information was downloaded from the TCGA database, such as survival time, survival status, age, gender, tumor grade, TNM stage. And then, the patients with incomplete clinical information, survival time that less than 30 days and nonhepatocellular carcinoma were removed. Since all of these data involved in this study were publicly available, the ethics committee has no specific ethical approval.

\subsection{Screening of Autophagy-Related IncRNAs}

A list of the autophagy-related genes was downloaded from the Human Autophagy Database (HADb, http://www.autophagy.lu/). The autophagy gene expression profile was extracted from the fore mentioned protein-coding gene profile data. To identify the potential IncRNA related to autophagy-related genes, we performed a Pearson correlation analysis in the IncRNA and autophagy-related gene expression profile. The thresholds were set as follows: $|\mathrm{R}|>0.4$ and $\mathrm{P}<0.001$ were considered a strong correlation.

\subsection{Identify prognosis-related autophagy IncRNAs and Calculate the Risk Score}

To confirm the potential prognostic value of autophagy-related IncRNAs, univariate Cox regression analysis and product-limit method (Kaplan-Meier method) were used to assess the association between autophagy-related IncRNA expression and survival data. Those autophagy-related IncRNAs are significantly related to survival (Both Kaplan-Meier method and univariate Cox regression satisfy $P$ value $<0.01$ ) were obtained as prognosis-related IncRNAs. Those autophagy-related IncRNAs were then used into multivariate Cox regression analysis to obtain regression coefficients $(\beta)$ with the lowest Akaike information criterion (AIC) values and then establish a risk score. The risk score calculation based as follows $=\beta$ IncRNA1 $\times$ ExpressionlncRNA1 + $\mid$ IncRNA2 $\times$ ExpressionlncRNA2 $+\ldots$ $+\beta I n c R N A 1 n \times$ ExpressionIncRNAn. According to the median risk score, the patients were classified into high-risk and low-risk groups. The two groups were assessed using the log-rank test.

\subsection{Analysis of Risk Score Model}

The clinical data and risk score utilized univariate and multivariate Cox regression analysis for evaluating whether the risk score of the autophagy-Related IncRNAs can be an independent indicator for the prognosis. And then, the receiver operating characteristic (ROC) curve and area under the ROC curve (AUC value) were performed to evaluate diagnostic efficacies. Moreover, it assessed the correlation between risk score and clinical data.

\subsection{Functional Analysis}

To further explore the Gene Ontology (GO) and the Kyoto Encyclopedia of Genes and Genomes (KEGG) pathways, these prognostic autophagy-related IncRNAs may participate. We performed Pearson correlation analysis in the final prognostic autophagy-related IncRNA and protein-coding gene profile data. The thresholds were set as follows: $|\mathrm{R}|>0.4$ and $\mathrm{P}<0.001$. Then we analyzed those coexpressed genes with prognostic autophagy-related IncRNAs through the clusterProfiler package to speculate the pathways and biological processes that IncRNA may participate in.

\section{Results}

\subsection{Acquisition of Autophagy-related IncRNAs}

A total of 223 patients with complete clinical information, 14,748 IncRNAs, and 19,767 mRNA were screened from the TCGA-HCC, 232 autophagy genes were obtained from HADb, among which 213 genes were expressed in TCGA-HCC. And then, 557 autophagy-related IncRNAs were identified according to the Pearson correlation analysis (Table S1).

\subsection{The Prognostic Autophagy-Related IncRNA}

According to univariate Cox regression analysis and product-limit method (P value $<0.01), 39$ autophagy-related IncRNAs which related to prognostic have been selected (Table S2). Subsequently, seven autophagy-related IncRNAs that had a co-expression relationship with 65 autophagy genes were identified as robust independent prognostic factors after Multivariate Cox regression (Figure 1). Among them, 
PRRT3-AS1 (HR 1.1028, 95\%Cl 1.0284-1.1827), RP11-479G22.8 (HR 1.0936, 95\%Cl 1.0261-1.1656), RP11-73M18.8 (HR 1.0635, 95\%Cl 1.0070-1.1233), LINC01138 (HR 1.2752, 95\%Cl 1.0110-1.6085), CTD-2510F5.4 (HR 1.1355, 95\%Cl 1.0607-1.2157), and RP11-324I22.4 (HR 1.2956, 95\% Cl 1.0181-1.6488) were unfavorable prognostic factors, and the CTC-297N7.9 (HR 0.577, 95\%Cl 0.3363-0.9900) was beneficial prognostic factor (Figure 2 and Table 1). According to the Kaplan-Meier overall survival (OS) curves, The OS of the high-risk group for HCC patients was significantly shorter than the low-risk group ( $\mathrm{P}=2.292 \mathrm{e}-10)$ (Figure 3A). The five years survival rate of the high-risk group ( $\mathrm{HR} 0.286,95 \% \mathrm{Cl} 0.199-0.411)$ is less than half of the low-risk group (HR 0.694, 95\% $\mathrm{Cl} 0.547-0.77$ ). Besides, all HCC patients' risk scores were ranked to analyze the distributions of the autophagy-related IncRNAs (Figure 3D). The distributions of survival status indicated that the high-risk group's survival rate and time were significantly decreased compared to the low-risk group (Figure 3C). Similarly, the expression of 6 unfavorable prognostic factors (PRRT3-AS1, RP11-479G22.8, RP11-73M18.8, LINC01138, CTD2510F5.4, and RP11-324I22.4) was increased with the risk score increases; on the contrary, the expression of beneficial prognostic factor (CTC-297N7.9) was decreased (Figure 3B).

\subsection{Clinical Value of the Prognostic Autophagy-Related IncRNA}

To evaluate whether the seven autophagy-related IncRNAs could be used as the independent prognosis biomarkers of patients in HCC, Univariate cox regression analysis, and Multivariate cox regression analysis were utilized to evaluate the relationship between the clinical data and risk score. Univariate Cox regression indicated that risk score $(P<0.001,95 \% \mathrm{Cl} 1.210-1.389), T(P<0.001,95 \% \mathrm{Cl} 1.443-$ $2.287)$, and stage $(P<0.001,95 \% \mathrm{Cl} 1.466-2.408)$ were independent prognostic indicators (Figure $4 \mathrm{~A}$ and Table 2). However, after implying multivariate analysis, only the risk score remains the independent prognostic indicator $(\mathrm{P}<0.001,95 \% \mathrm{Cl} 1.197-1.400)$ (Figure $4 \mathrm{~B}$ and Table 2). Next, the receiver operating characteristic (ROC) curves were utilized to evaluate the risk score's predictive performance. The area under the ROC (AUC) curve of 1-, 3-, and 5-year were $0.853,0.794$, and 0.764 , respectively (Figure $4 C$ ). These findings signified that the risk score could be a good factor in predicting HCC patients' prognosis. The risk score increased with stage, and T showed that those autophagy-related IncRNAs might be associated with the progression of HCC (Table 3).

\subsection{Functional Analysis}

Under the inclusion criteria of $|\mathrm{R}|>0.4$ and $\mathrm{P}<0.001$, a total of 3580 genes that have a co-expression relationship with 7 prognostic autophagy-related IncRNAs were obtained (Table S3). KEGG analysis shows that the 7 IncRNAs are directly or indirectly involved in Spliceosome, Cell cycle, RNA transport, DNA replication, Ribosome, mRNA surveillance pathway, and Endocytosis (Figure 5B). GO results indicate that these IncRNAs may be related to the biological process of RNA splicing, mRNA splicing, RNA localization, covalent chromatin modification, and histone modification (Figure 5A).

\section{Discussion}

Autophagy, an evolutionary and conservative multistage lysosomal degradation process that promotes metabolism and healthy circulation, plays a complex and contradictory role in tumor formation and cancer treatment [12]. As a subclass of the ncRNAs family, IncRNAs play an indispensable role in various biological processes of tumorigenesis, which are considered a new type of biomarker for cancer diagnosis and prognosis widely concerned [31]. The current researches were mainly focused on the function of single or a few IncRNAs involved in autophagy in HCC patients [32-34]. Therefore, it is necessary to explore more autophagy-related IncRNAs to predict HCC patients' prognosis.

In our study, autophagy-related IncRNAs were obtained by establishing the co-expression network of IncRNAs and autophagy genes. Univariate and multivariate Cox regression analyses were used to obtain the 7 prognostic autophagy-related IncRNAs, including PRRT3AS1, RP11-479G22.8, RP11-73M18.8, LINC01138, CTD-2510F5.4, and RP11-324I22.4, and CTC-297N7.9.The seven autophagy-related IncRNAs may become prognostic molecular markers and potential therapeutic targets for HCC patients for follow-up research.

On the one hand, as the only beneficial prognostic IncRNA in the prognostic prediction model. ,the gene alias of CTC-297N7.9 is IncTMEM220-1, which is an intergenic ncRNA. An HCC study showed that CTC-297N7.9 might be related to cofactor/chromatin/NAD binding and oxidoreductase/DNA-dependent ATPase activity [35]. Besides, due to the specific low expression and high methylation of TMEM220 in gastric cancer tissues [36], some scholars speculate that CTC-297N7.9 that located upstream of the protein-coding gene TMEM220, may be able to regulate the methylation of TMEM220 or participate in autophagy through its functional proteins, which in turn affects the prognosis of HCC patients [37]. In our research, we have speculated that the highly expressed CTC-297N7.9 may be an inhibitory factor in the progression of HCC. This speculation was confirmed in another study on liver cancer, and the high expression of 
CTC-297N7.9 often predicts better overall survival and disease-free survival [38] and indicates that CTC-297N7.9 may be one of the critical molecules to improve HCC patients' survival, and it can be further explored in subsequent studies on HCC.

On the other hand, the 6 unfavorable prognostic IncRNAs in the prognostic prediction model have also been attached to various cancers. The official full name of PRRT3-AS1 is PRRT3 antisense RNA 1, as a non-protein-coding RNA, which is mainly expressed in liver tissue (RPKM 0.15), fat (RPKM 4.4), prostate (RPKM 3.3), and brain tissue (RPKM 3.0) [39]. In prostate cancer, Fan et al. confirmed that PRRT3-AS1 has a targeting relationship with PPARY. Its silence can promote apoptosis autophagy and inhibit the proliferation, migration, and invasion of tumor cells through the mTOR signaling pathway [40]. Besides, PRRT3-AS1 is also considered to be related to GBM patients' prognosis [41]. RP11-479G22.8 is also known as Inc-ITGB1-1 in the LNCipedia database [42], and its transcription size is $2051 \mathrm{bp}$. Through the IncRNA disease prediction module of the IncRNASNP2 database [43], RP11-479G22.8 is closely related to HCC (P $<0.001$ ). Therefore, RP11-479G22.8 is expected to be one of the potential indicators for prognostic prediction in HCC patients [35]. RP11-73M18.8 is a sense-intronic IncRNA with a transcript size of $811 \mathrm{bp}$, also known as Inc-ZFYVE21-3. Sense-intronic IncRNA is a sequence in the intron of the coding gene on the sense strand. It might harbor different histone modification at the transcription start site (TSS) than other ncRNAs [44], which indicated that these intronic IncRNAs maybe the novel biomarkers, such as type 2 diabetes mellitus [45]. LINC01138 is also a member of the sense intron ncRNA, located on chr1. Its abnormal expression has an important influence on the occurrence and development of several cancers. In prostate cancer (PCa), as a IncRNA that directly target AR, the high expression of LINC01138 can promote the proliferation of tumor cells and inhibit their apoptosis, which indicated that LINC01138 could be a diagnostic and prognostic marker for PCa [46]. Besides, LINC01138 can increase the arginine methylation and protein stability of sterol regulatory element-binding protein one by interacting with PRMT5, thereby promoting lipid desaturation and cell proliferation in clear cell renal cell carcinoma and being associated with poor prognosis[47]. However. The knockdown of LINC01138 can inhibit the viability, proliferation, invasion, and migration and promotes apoptosis of gastric cancer cells through the LINC01138/miR-1273e/MAPK axis [48].In some studies related to HCC, high-expressed LINC01138 is not only significantly associated with poor survival [49] but also can interact with arginine methyltransferase 5 to promote cell proliferation, tumorigenicity, tumor invasion, and metastasis [50]. CTD2510F5.4 is a 321 bp antisense IncRNA, also known as Inc-SKA2-1 in the LNCipedia database. Through the NPInter v4.0 database [51], we found that CTD-2510F5.4 mainly interacts with genes in the mRNA surveillance pathway and RNA transport pathway. The high expressed CTD-2510F5.4 also has a significant co-expression relationship with mRNAs of the cell cycle, DNA replication, and p53 signaling pathway [52]. It is closely related to the poor prognosis of patients with lung adenocarcinoma[53]. In gastric cancer, the highly expressed CTD-2510F5.4 may be an independent risk factor for tumors with pathological grade < III and no vascular or nerve infiltration [54]. RP11-324I22.4 is an antisense IncRNA; the gene alias is Inc-CUL2-3. As cancer or tumor suppressor genes, antisense IncRNAs play an essential role in the occurrence and development of human cancer[55-57]. Although there is currently no disease research related to RP11-324I22.4, antisense IncRNAs may certainly be the promising tumor biomarker and therapeutic target in future research.

The risk scoring model we identified ( AUC of $1-, 3$-, and 5 -year were $0.853,0.794$, and 0.764 , respectively ) is more reliable than a similar study in HCC (AUC of the 1-, 3-, and 5-year survival are $0.764,0.738$, and 0.717 , respectively) [58], but it still has its limitations. This study is only based on the TCGA database, and there are no suitable datasets in other databases to verify the risk prediction model. Furthermore, the research is only conducted at the level of bioinformatics; a comprehensive in vitro experiment is needed further to explore the regulatory mechanism of these autophagy IncRNAs.

\section{Conclusions}

In general, the constructed risk prediction model of autophagy-related IncRNAs (PRRT3-AS1, RP11-479G22.8, RP11-73M18.8, LINC01138, CTD-2510F5.4, and RP11-324I22. 4, and CTC-297N7.9) based on autophagy genes is robust and promising. The IncRNAs in this model can be used as potential biomarkers for the prognosis of HCC, which will help the individualized treatment of HCC.

\section{Declarations}

\section{Author's contributions}

Liu Yang and Yaping Zhou contribute equally. Liu Yang and Yaping Zhou contributed to the conception and design of the study. Liu Yang and Xiangxin Zhang organized the database and statistical analysis. Liu Yang wrote the first draft of the manuscript. Xiaotong Zhao contributed to manuscript revision. Jianfeng Fu and Lu Wang contributed to the manuscript read and approved the submitted version. 


\section{Funding}

The design, the collection, analysis, interpretation of data, and the writing of the manuscript were supported in part by the special scientific research project of health young medical science and technology talents in Xinjiang Uygur Autonomous Region (WYWY202010).

\section{Availability of data and materials}

All of these data involved in this study were publicly available.

\section{Ethics approval and consent to participate}

Not applicable.

\section{Consent for publication}

Not applicable.

\section{Competing interests}

The authors declare that they have no competing interests.

\section{Contributor Information}

Liu Yang, Email: 2295722106@qq.com.

Yaping Zhou, Email: 1462755959@qq.com

\section{Acknowledgments}

Not applicable.

\section{References}

1. Berndt N, Eckstein J, Heucke N, Gajowski R, Stockmann M, Meierhofer D, Holzhütter HG. Characterization of Lipid and Lipid Droplet Metabolism in Human HCC, 2019. Cells. 2019;8(5): undefined. doi:10.3390/cells8050512.

2. Bray F, Ferlay J, Soerjomataram I, Siegel RL, Torre LA, Jemal A. Global cancer statistics 2018: GLOBOCAN estimates of incidence and mortality worldwide for 36 cancers in 185 countries, 2018. CA Cancer J Clin. 2018;68(6):394-424. doi:10.3322/caac.21492.

3. Pei Y, Zhang T, Renault V, Zhang X. An overview of hepatocellular carcinoma study by omics-based methods, 2009. Acta Biochim Biophys Sin (Shanghai). 2019;41(1):1-15. doi:10.1093/abbs/gmn001.

4. Fitzmorris P, Shoreibah M, Anand BS, Singal AK. Management of hepatocellular carcinoma, 2015. Management of hepatocellular carcinoma. J Cancer Res Clin Oncol. 2015;141(5):861-76. doi:10.1007/s00432-014-1806-0.

5. Chen S, Cao Q, Wen W, Wang H. Targeted therapy for hepatocellular carcinoma: Challenges and opportunities, 2019. Cancer Lett.2019;460(undefined): 1-9. doi:10.1016/j.canlet.2019.114428.

6. Zheng T, Xie F, Geng L, Sui CJ, Dai DH, Shen RX, Yan L, Yang JM. Safety and long-term outcomes of anatomic left hepatic trisectionectomy for intermediate and advanced hepatocellular carcinoma, 2015. J Gastroenterol Hepatol. 2019;30(6): 1015-23. doi:10.1111/jgh.12887.

7. Tian M, Zhao B, Martin FL, Morais CLM, Liu L, Huang Q, Zhang J, Shen H. Gene-environment interactions between GSTs polymorphisms and targeted epigenetic alterations in hepatocellular carcinoma following organochlorine pesticides (OCPs) exposure, 2020. Environ Int.2020;134(undefined): 105313. doi:10.1016/j.envint.2019.105313.

8. Li WT, Zou AE, Honda CO, Zheng H, Wang XQ, Kisseleva T, Chang EY, Ongkeko WM. Etiology-Specific Analysis of Hepatocellular Carcinoma Transcriptome Reveals Genetic Dysregulation in Pathways Implicated in Immunotherapy Efficacy, 2019. Cancers (Basel). 2019; 11(9): undefined. doi:10.3390/cancers11091273.

9. Lim LJ, Wong SYS, Huang F, Lim S, Chong SS, Ooi LL, Kon OL, Lee CG. Roles and Regulation of Long Noncoding RNAs in Hepatocellular Carcinoma, 2019. Cancer Res. 2019; 79(20): 5131-5139. doi:10.1158/0008-5472.CAN-19-0255 
10. Harris WP, Wong KM, Saha S, Dika IE, Abou-Alfa GK. Biomarker-Driven and Molecular Targeted Therapies for Hepatobiliary Cancers, 2018. Semin Oncol. 2018; 45(3): 116-123. doi:10.1053/j.seminoncol.2018.03.002.

11. Amaravadi RK, Kimmelman AC, Debnath J. Targeting Autophagy in Cancer: Recent Advances and Future Directions, 2019. Cancer Discov. 2019; 9(9): 1167-1181. doi:10.1158/2159-8290.CD-19-0292.

12. Levine B, Kroemer G. Biological Functions of Autophagy Genes: A Disease Perspective, 2019. Cell.2019; 176(null): 11-42. doi:10.1016/j.cell.2018.09.048.

13. Towers CG, Wodetzki D, Thorburn A. Autophagy and cancer: Modulation of cell death pathways and cancer cell adaptations, 2020. J Cell Biol.2020; 219(1): undefined. doi:10.1083/jcb.201909033

14. Zhu XG, Chudnovskiy A, Baudrier L, Prizer B, Liu Y, Ostendorf BN, Yamaguchi N, Arab A, Tavora B, Timson R, et al. Functional Genomics In Vivo Reveal Metabolic Dependencies of Pancreatic Cancer Cells, 2020. Cell Metab. 2020; undefined(undefined): undefined. doi:10.1016/j.cmet.2020.10.017.

15. Sharifi MN, Mowers EE, Drake LE, Collier C, Chen H, Zamora M, Mui S, Macleod KF. Autophagy Promotes Focal Adhesion Disassembly and Cell Motility of Metastatic Tumor Cells through the Direct Interaction of Paxillin with LC3, 2016. Cell Rep. 2016; 15(8): 1660-72. doi:10.1016/j.celrep.2016.04.065.

16. Fan Q, Yang L, Zhang X, Ma Y, Li Y, Dong L, Zong Z, Hua X, Su D, Li H, et al. Autophagy promotes metastasis and glycolysis by upregulating MCT1 expression and Wnt/ $\beta$-catenin signaling pathway activation in hepatocellular carcinoma cells, 2018. J Exp Clin Cancer Res.2018; 37(1): 9. doi:10.1186/s13046-018-0673-y.

17. Li J, Yang B, Zhou Q, Wu Y, Shang D, Guo Y, Song Z, Zheng Q, Xiong J. Autophagy promotes hepatocellular carcinoma cell invasion through activation of epithelial-mesenchymal transition, 2013. Carcinogenesis. 2013; 34(6): 1343-51. doi:10.1093/carcin/bgt063.

18. Evans JR, Feng FY, Chinnaiyan AM. The bright side of dark matter: IncRNAs in cancer, 2016. J Clin Invest. 2016; 126(8): $2775-82$. doi:10.1172/JCl84421.

19. Mercer TR, Dinger ME, Mattick JS. Long non-coding RNAs: insights into functions, 2009. Nat Rev Genet. 2009; 10(3): 155-9. doi:10.1038/nrg2521.

20. Beermann J, Piccoli MT, Viereck J, Thum T. Non-coding RNAs in Development and Disease: Background, Mechanisms, and Therapeutic Approaches, 2016. Physiol Rev. 2016; 96(4): 1297-325. doi:10.1152/physrev.00041.2015.

21. Prensner JR, Chinnaiyan AM. The emergence of IncRNAs in cancer biology, 2011. Cancer Discov. 2011; 1(5): 391-407. doi:10.1158/2159-8290.CD-11-0209.

22. Galvão MLTC, Coimbra EC. Long noncoding RNAs (IncRNAs) in cervical carcinogenesis: New molecular targets, current prospects, 2020. Crit Rev Oncol Hematol. 2020; 156(undefined): 103111. doi:10.1016/j.critrevonc.2020.103111.

23. Wang G, Feng B, Niu Y, Wu J, Yang Y, Shen S, Guo Y, Liang J, Guo W, Dong Z. A novel long noncoding RNA, LOC440173, promotes the progression of esophageal squamous cell carcinoma by modulating the miR-30d-5p/HDAC9 axis and the epithelialmesenchymal transition, 2020. Mol Carcinog. 2020; 59(12): 1392-1408. doi:10.1002/mc.23264.

24. Zhao M, Xin XF, Zhang JY, Dai W, Lv TF, Song Y. LncRNA GMDS-AS1 inhibits lung adenocarcinoma development by regulating miR96-5p/CYLD signaling, 2020. Cancer Med. 2020; 9(3): 1196-1208. doi:10.1002/cam4.2776.

25. Dower CM, Wills CA, Frisch SM, Wang HG. Mechanisms and context underlying the role of autophagy in cancer metastasis, 2018. Autophagy. 2018; 14(7): 1110-1128. doi:10.1080/15548627.2018.1450020.

26. Zhang Z, Li H, Hu Y, Wang F. Long non-coding RNA ADAMTS9-AS1 exacerbates cell proliferation, migration, and invasion via triggering of the PI3K/AKT/mTOR pathway in hepatocellular carcinoma cells, 2020. Am J Transl Res. 2020; 12(9): 5696-5707.

27. Peng N, He J, Li J, Huang H, Huang W, Liao Y, Zhu S. Long noncoding RNA MALAT1 inhibits the apoptosis and autophagy of hepatocellular carcinoma cell by targeting the microRNA-146a/PI3K/Akt/mTOR axis, 2020. Cancer Cell Int. 2020区 20(undefined): 165. doi:10.1186/s12935-020-01231-w.

28. Zhang W, Liu Y, Fu Y, Han W, Xu H, Wen L, Deng Y, Liu K. Long non-coding RNA LINC00160 functions as a decoy of microRNA-132 to mediate autophagy and drug resistance in hepatocellular carcinoma via inhibition of PIK3R3. 2020. Cancer Lett. 2020; 478(undefined): 22-33. doi:10.1016/j.canlet.2020.02.014.

29. Derrien T, Johnson R, Bussotti G, Tanzer A, Djebali S, Tilgner H, Guernec G, Martin D, Merkel A, Knowles DG, et al. The GENCODE v7 catalog of human long noncoding RNAs: analysis of their gene structure, evolution, and expression, 2012. Genome Res. 2012; 22(9): 1775-89. doi:10.1101/gr.132159.111. 
30. Yan X, Hu Z, Feng Y, Hu X, Yuan J, Zhao SD, Zhang Y, Yang L, Shan W, He Q, et al. Comprehensive Genomic Characterization of Long Non-coding RNAs across Human Cancers, 2015. Cancer Cell. 2015; 28(4): 529-540. doi:10.1016/j.ccell.2015.09.006.

31. Zhao Y, Guo Q, Chen J, Hu J, Wang S, Sun Y. Role of long non-coding RNA HULC in cell proliferation, apoptosis and tumor metastasis of gastric cancer: a clinical and in vitro investigation, 2014. Oncol Rep. 2014; 31(1): 358-64. doi:10.3892/or.2013.2850.

32. Chen CL, Tseng YW, Wu JC, Chen GY, Lin KC, Hwang SM, Hu YC. Suppression of hepatocellular carcinoma by baculovirus-mediated expression of long non-coding RNA PTENP1 and MicroRNA regulation, 2015. Biomaterials. 2015;44(undefined): 71-81. doi:10.1016/j.biomaterials.2014.12.023.

33. Yang L, Zhang X, Li H, Liu J. The long noncoding RNA HOTAIR activates autophagy by upregulating ATG3 and ATG7 in hepatocellular carcinoma, 2016. Mol Biosyst. 2016; 12(8): 2605-12. doi:10.1039/c6mb00114a.

34. Wu Y, Wang PS, Wang BG, Xu L, Fang WX, Che XF, Qu XJ, Liu YP, Li Z. Genomewide identification of a novel six-LncRNA signature to improve prognosis prediction in resectable hepatocellular carcinoma, 2018. Cancer Med. 2018; 7(12): 6219-6233. doi:10.1002/cam4.1854.

35. Choi B, Han TS, Min J, Hur K, Lee SM, Lee HJ, Kim YJ, Yang HK. MAL and TMEM220 are novel DNA methylation markers in human gastric cancer, 2017. Biomarkers. 2017; 22(1): 35-44. doi:10.1080/1354750X.2016.1201542.

36. Wang Z, Wu Q, Feng S, Zhao Y, Tao C. Identification of four prognostic LncRNAs for survival prediction of patients with hepatocellular carcinoma, 2017. PeerJ. 2017; 5(undefined): e3575. doi:10.7717/peerj.3575.

37. Zhu S, Huang X, Zhang K, Tan W, Lin Z, He Q, Chen Y, Shang C. Low expression of long noncoding RNA CTC-297N7.9 predicts poor prognosis in patients with hepatocellular carcinoma, 2019. Cancer Med. 2019; 8(18): 7679-7692. doi:10.1002/cam4.2618.

38. Fagerberg L, Hallström BM, Oksvold P, Kampf C, Djureinovic D, Odeberg J, Habuka M, Tahmasebpoor S, Danielsson A, Edlund K, et al. Analysis of the human tissue-specific expression by genome-wide integration of transcriptomics and antibody-based proteomics, 2014. Mol Cell Proteomics. 2014; 13(2): 397-406. doi:10.1074/mcp.M113.035600.

39. Fan L, Li H, Wang W. Long non-coding RNA PRRT3-AS1 silencing inhibits prostate cancer cell proliferation and promotes apoptosis and autophagy, 2020. Exp Physiol. 2020; 105(5): 793-808. doi:10.1113/EP088011.

40. Liang R, Zhi Y, Zheng G, Zhang B, Zhu H, Wang M. Analysis of long non-coding RNAs in glioblastoma for prognosis prediction using weighted gene co-expression network analysis, Cox regression, and L1-LASSO penalization, 2019. Onco Targets Ther. 2019; 12(undefined): 157-168. doi:10.2147/OTT.S171957.

41. Volders PJ, Anckaert J, Verheggen K, Nuytens J, Martens L, Mestdagh P, Vandesompele J. LNCipedia 5: towards a reference set of human long non-coding RNAs, 2019. Nucleic Acids Res. 2019; 47(null): D135-D139. doi:10.1093/nar/gky1031.

42. Miao YR, Liu W, Zhang Q, Guo AY. IncRNASNP2: an updated database of functional SNPs and mutations in human and mouse IncRNAs, 2018. Nucleic Acids Res. 2018; 46(null): D276-D280. doi:10.1093/nar/gkx1004.

43. Ghosh S, Sati S, Sengupta S, Scaria V. Distinct patterns of epigenetic marks and transcription factor binding sites across promoters of sense-intronic long noncoding RNAs, 2105. J Genet. 2015; 94(1): 17-25. doi:10.1007/s12041-015-0484-2.

44. Anbari DM, Al-Harithy RN. Inc-GHRL-3:2Ghrelin intronic IncRNAs, and, as novel biomarkers in type 2 diabetes mellitus, 2020. Arch Physiol Biochem. 2020; undefined(undefined): 1-5. doi:10.1080/13813455.2020.1817095.

45. Wan X, Huang W, Yang S, Zhang Y, Pu H, Fu F, Huang Y, Wu H, Li T, Li Y. Identification of androgen-responsive IncRNAs as diagnostic and prognostic markers for prostate cancer, 2016. Oncotarget. 2016; 7(37): 60503-60518. doi:10.18632/oncotarget.11391.

46. Zhang X, Wu J, Wu C, Chen W, Lin R, Zhou Y, Huang X. The LINC01138 interacts with PRMT5 to promote SREBP1-mediated lipid desaturation and cell growth in clear cell renal cell carcinoma, 2018. Biochem Biophys Res Commun. $2018 ; 507$ (null): $337-342$. doi:10.1016/j.bbrc.2018.11.036.

47. Dou GX, Zhang JN, Wang P, Wang JL, Sun GB. Long Intergenic Non-Protein-Coding RNA 01138 Accelerates Tumor Growth and Invasion in Gastric Cancer by Regulating miR-1273e, 2019. Med Sci Monit. 2019; 25(undefined): 2141-2150. doi:10.12659/MSM.914248.

48. Jiang H, Shi X, Ye G, Xu Y, Xu J, Lu J, Lu W. Up-regulated long non-coding RNA DUXAP8 promotes cell growth through repressing Krüppel-like factor 2 expression in human hepatocellular carcinoma, 2019. Onco Targets Ther. 2019; 12 (undefined): 7429 -7436. doi:10.2147/OTT.S214336.

49. Li Z, Zhang J, Liu X, Li S, Wang Q, Hu Z, Yu T, Ding J, Li J, Yao M, et al. The LINC01138 drives malignancies via activating arginine methyltransferase 5 in hepatocellular carcinoma, 2018. Nat Commun. 2018; 9(1):1572. doi:10.1038/s41467-018-04006-0. 
50. Teng X, Chen X, Xue H, Tang Y, Zhang P, Kang Q, Hao Y, Chen R, Zhao Y, He S. NPInter v4.0: an integrated database of ncRNA interactions, 2020. Nucleic Acids Res. 2020; 48(null): D160-D165. doi:10.1093/nar/gkz969.

51. Wang Y, Fu J, Wang Z, Lv Z, Fan Z, Lei T. Screening key IncRNAs for human lung adenocarcinoma based on machine learning and weighted gene co-expression network analysis, 2019. Cancer Biomark. 2019; 25(4): 313-324. doi:10.3233/CBM-190225.

52. Wang X, Li G, Luo Q, Xie J, Gan C. Integrated TCGA analysis implicates IncRNA CTB-193M12.5 as a prognostic factor in lung adenocarcinoma, 2018. Cancer Cell Int. 2018; 18(undefined): 27. doi:10.1186/s12935-018-0513-3.

53. Wang Z, Qin B. Prognostic and clinicopathological significance of long noncoding RNA CTD-2510F5.4 in gastric cancer, 2019. Gastric Cancer. 2019; 22(4): 692-704. doi:10.1007/s10120-018-00911-x.

54. Da CM, Cheng ZY, Gong CY, Nan W, Zhou KS, Zhao GH, Zhang HH. Role of HAND2-AS1 in human tumors, 2020. Clin Chim Acta. 2010; 511(undefined): 189-197. doi:10.1016/j.cca.2020.10.020.

55. Ruan Z, Deng H, Liang M, Xu Z, Lai M, Ren H, Deng X, Su X. Downregulation of long non-coding RNA MAFG-AS1 represses tumorigenesis of colorectal cancer cells through the microRNA-149-3p-dependent inhibition of HOXB8, 2020. Cancer Cell Int. 2020; 20(undefined): 511. doi:10.1186/s12935-020-01485-4.

56. Yang J, Zou Y, Wu J, Chen B, Luo C, Chen X, Shen H, Luo L. ZEB2-AS1The Long Noncoding RNA Contributes to Proliferation and Epithelial-to-Mesenchymal Transition of Osteosarcoma, 2020. Cancer Biother Radiopharm. 2020; undefined(undefined): undefined. doi:10.1089/cbr.2019.3433.

57. Wu H, Liu T, Qi J, Qin C, Zhu Q. Four Autophagy-Related IncRNAs Predict the Prognosis of HCC through Coexpression and ceRNA Mechanism, 2020. Biomed Res Int.2020; 2020(undefined): 3801748. doi:10.1155/2020/3801748.

\section{Tables}

Table 1

The HR, 95\% Cl of HR, and P-value of the 7 autophagyrelated IncRNA based on the multivariate Cox regression analysis. HR, hazard ratio.

\begin{tabular}{|llll|}
\hline Name & HR & $95 \%$ Cl of HR & P-value \\
\hline PRRT3-AS1 & 1.1028 & $1.0284-1.1827$ & 0.0061 \\
\hline RP11-479G22.8 & 1.0936 & $1.0261-1.1656$ & 0.0059 \\
\hline RP11-73M18.8 & 1.0635 & $1.0070-1.1233$ & 0.0272 \\
\hline LINC01138 & 1.2752 & $1.0110-1.6085$ & 0.0401 \\
\hline CTD-2510F5.4 & 1.1355 & $1.0607-1.2157$ & 0.0003 \\
\hline SNHG12 & 0.8353 & $0.7157-0.9747$ & 0.0223 \\
\hline CTC-297N7.9 & 0.577 & $0.3363-0.9900$ & 0.0459 \\
\hline RP11-324I22.4 & 1.2956 & $1.0181-1.6488$ & 0.0352 \\
\hline
\end{tabular}


Table 2

Clinical characteristics and risk scores based on Univariate and multiple Cox regression analysis.

\begin{tabular}{|lllllll|}
\hline \multicolumn{7}{|l}{ Univariate Cox regression analysis } \\
\hline Type & B & SE & $\mathbf{z}$ & HR & $95 \%$ Cl of HR & P-value \\
\hline Age & 0.007 & 0.009 & 0.707 & 1.007 & $0.989-1.025$ & 0.480 \\
\hline gender & 0.251 & 0.239 & 1.048 & 0.778 & $0.487-1.244$ & 0.295 \\
\hline Grade & 0.013 & 0.158 & 0.082 & 1.013 & $0.743-1.380$ & 0.934 \\
\hline Stage & 0.631 & 0.127 & 4.978 & 1.879 & $1.466-2.408$ & $<0.001$ \\
\hline T & 0.597 & 0.118 & 5.077 & 1.816 & $1.443-2.287$ & $<0.001$ \\
\hline M & 1.367 & 0.592 & 2.310 & 3.924 & $1.230-12.519$ & 0.021 \\
\hline N & 0.727 & 0.719 & 1.012 & 2.070 & $0.506-8.471$ & 0.312 \\
\hline Risk Score & 0.259 & 0.035 & 7.375 & 1.296 & $1.210-1.389$ & $<0.001$ \\
\hline Multiple Cox regression analysis & & & \\
\hline Type & $\mathbf{B}$ & $\mathbf{S E}$ & $\mathbf{z}$ & HR & $95 \%$ Cl of HR & P-value \\
\hline Age & 0.016 & 0.009 & 1.682 & 1.016 & $0.997-1.035$ & 0.093 \\
\hline gender & 0.104 & 0.274 & 0.380 & 0.901 & $0.527-1.542$ & 0.704 \\
\hline Grade & 0.119 & 0.167 & 0.712 & 1.127 & $0.811-1.564$ & 0.477 \\
\hline Stage & 0.043 & 0.519 & 0.083 & 0.958 & $0.346-2.647$ & 0.934 \\
\hline T & 0.540 & 0.466 & 1.160 & 1.717 & $0.684-4.278$ & 0.246 \\
\hline M & 0.467 & 0.683 & 0.683 & 1.595 & $0.418-6.087$ & 0.495 \\
\hline N & 0.759 & 0.943 & 0.804 & 2.135 & $0.336-13.553$ & 0.421 \\
\hline Risk Score & 0.258 & 0.040 & 6.470 & 1.295 & $1.197-1.400$ & $<0.001$ \\
\hline
\end{tabular}


Table 3

The influence of risk score on clinical characteristics of patients with HCC.

\begin{tabular}{|c|c|c|c|c|c|c|}
\hline Clinical & Group & $n$ & Mean & SD & $t$ & P-value \\
\hline \multirow[t]{2}{*}{ Age } & $<=65$ & 165 & 1.641 & 2.279 & \multirow[t]{2}{*}{1.516303} & \multirow[t]{2}{*}{0.131} \\
\hline & $>65$ & 70 & 1.253 & 1.54 & & \\
\hline \multirow[t]{2}{*}{ Gender } & Female & 74 & 1.494 & 2.008 & \multirow[t]{2}{*}{-0.16151} & \multirow[t]{2}{*}{0.872} \\
\hline & Male & 161 & 1.54 & 2.134 & & \\
\hline \multirow[t]{2}{*}{ Grade } & G1-2 & 132 & 1.414 & 2.143 & \multirow[t]{2}{*}{-0.85488} & \multirow[t]{2}{*}{0.394} \\
\hline & G3 & 93 & 1.655 & 2.031 & & \\
\hline \multirow[t]{2}{*}{ Stage } & Stage I-II & 163 & 1.184 & 1.337 & \multirow[t]{2}{*}{-2.95842} & \multirow[t]{2}{*}{0.004} \\
\hline & Stage III-IV & 72 & 2.299 & 3.074 & & \\
\hline \multirow[t]{2}{*}{$\mathbf{T}$} & T1-2 & 167 & 1.196 & 1.332 & \multirow[t]{2}{*}{-2.87851} & \multirow[t]{2}{*}{0.005} \\
\hline & T3-4 & 68 & 2.335 & 3.152 & & \\
\hline \multirow[t]{2}{*}{ M } & MO & 231 & 1.523 & 2.105 & \multirow[t]{2}{*}{-0.29115} & \multirow[t]{2}{*}{0.788} \\
\hline & M1 & 4 & 1.678 & 1.026 & & \\
\hline \multirow[t]{2}{*}{$\mathbf{N}$} & No & 231 & 1.521 & 2.104 & \multirow[t]{2}{*}{-0.38383} & \multirow[t]{2}{*}{0.725} \\
\hline & N1 & 4 & 1.769 & 1.259 & & \\
\hline
\end{tabular}

\section{Figures}
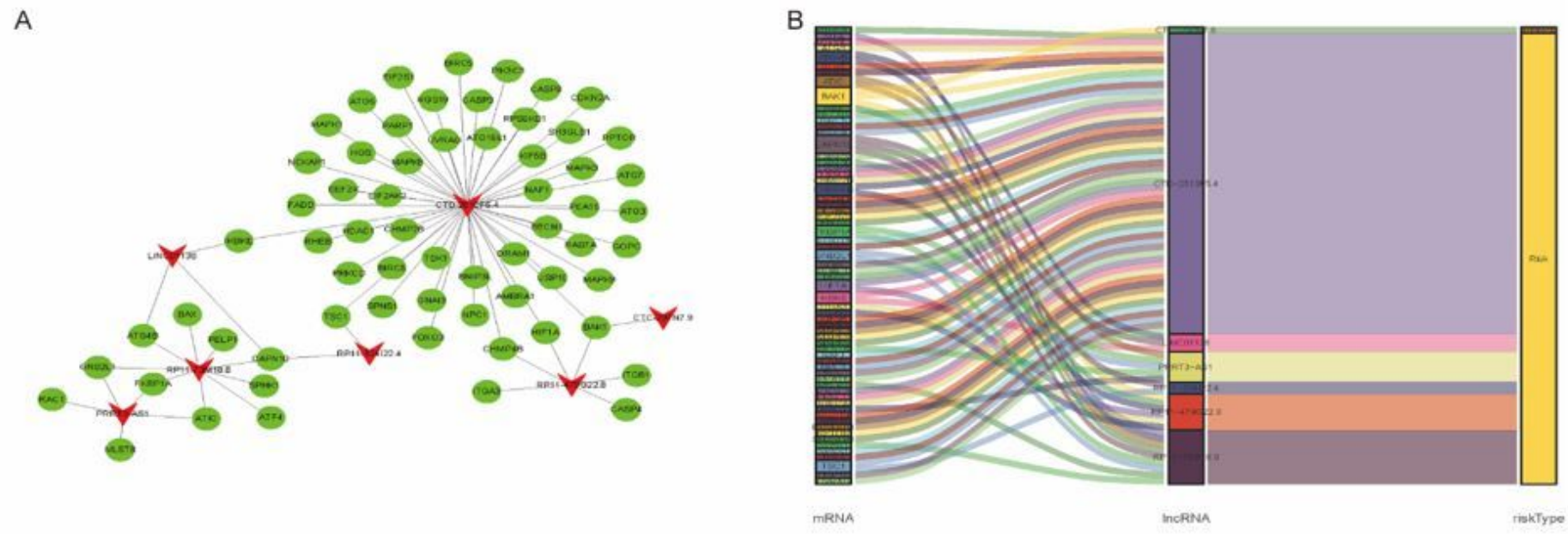

Figure 1

The Co-expression network and Sankey diagram of prognostic autophagy-related IncRNAs. (A) The Co-expression network between prognostic autophagy-related IncRNAs and autophagy-related genes in Hepatocellular carcinoma. The green circle nodes represent prognostic autophagy-related IncRNAs, and the red $V$ nodes represent autophagy-related genes. The Co-expression network was visualized using Cytoscape 3.7.2 software. (B) Sankey's diagram showed the relationship between prognostic autophagy-related IncRNAs, autophagy genes, and risk types. 

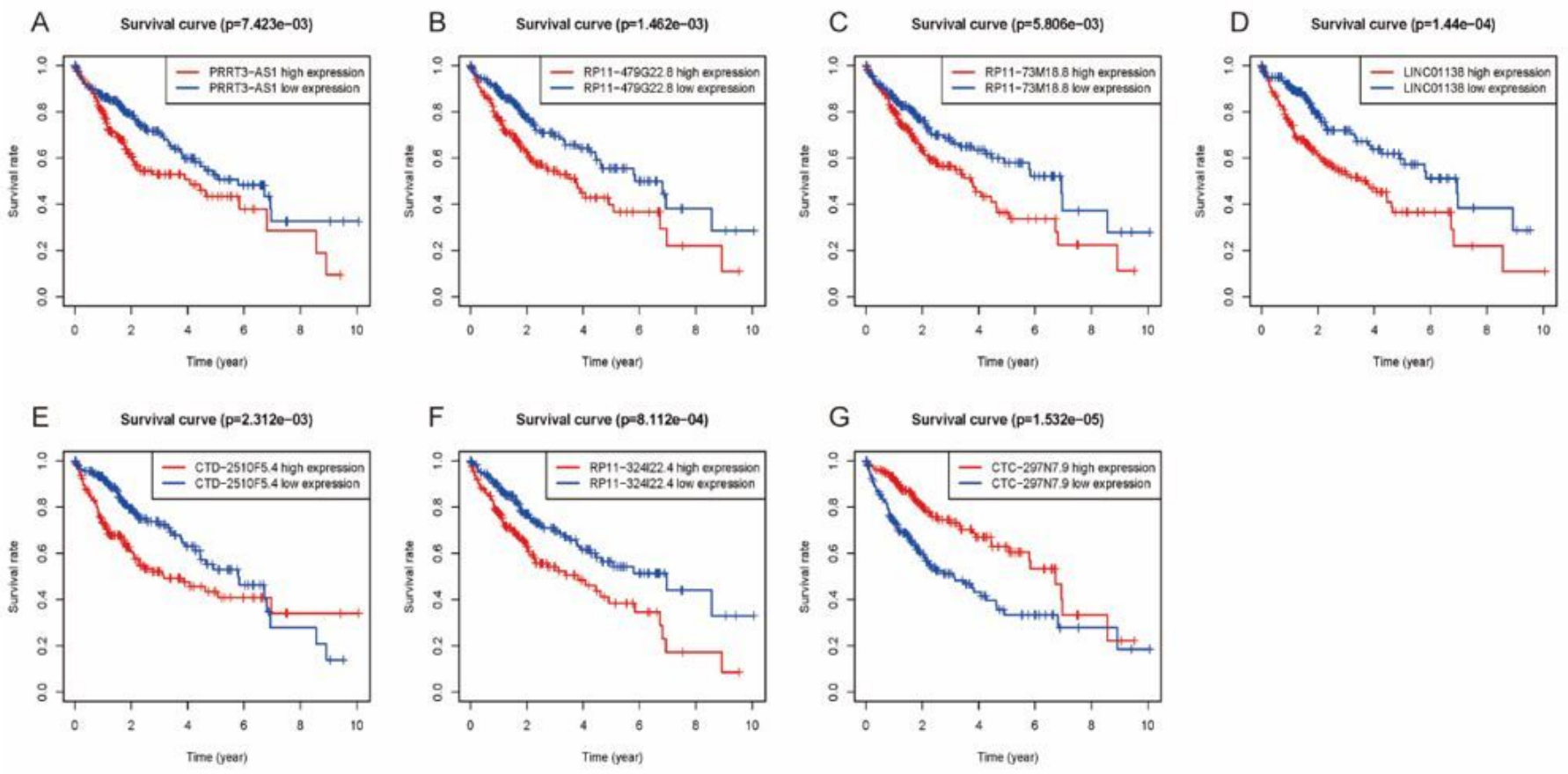

\section{Figure 2}

The KM survival curves of 7 prognostic autophagy-related IncRNAs. (A) PRRT3-AS1, (B) RP11-479G22.8, (C) RP11-73M18.8, (D) LINC01138, (E) CTD-2510F5.4, and (F) RP11-324I22.4 were harmful prognostic factors, and the (G) CTC-297N7.9 was a favorable prognostic factor. 

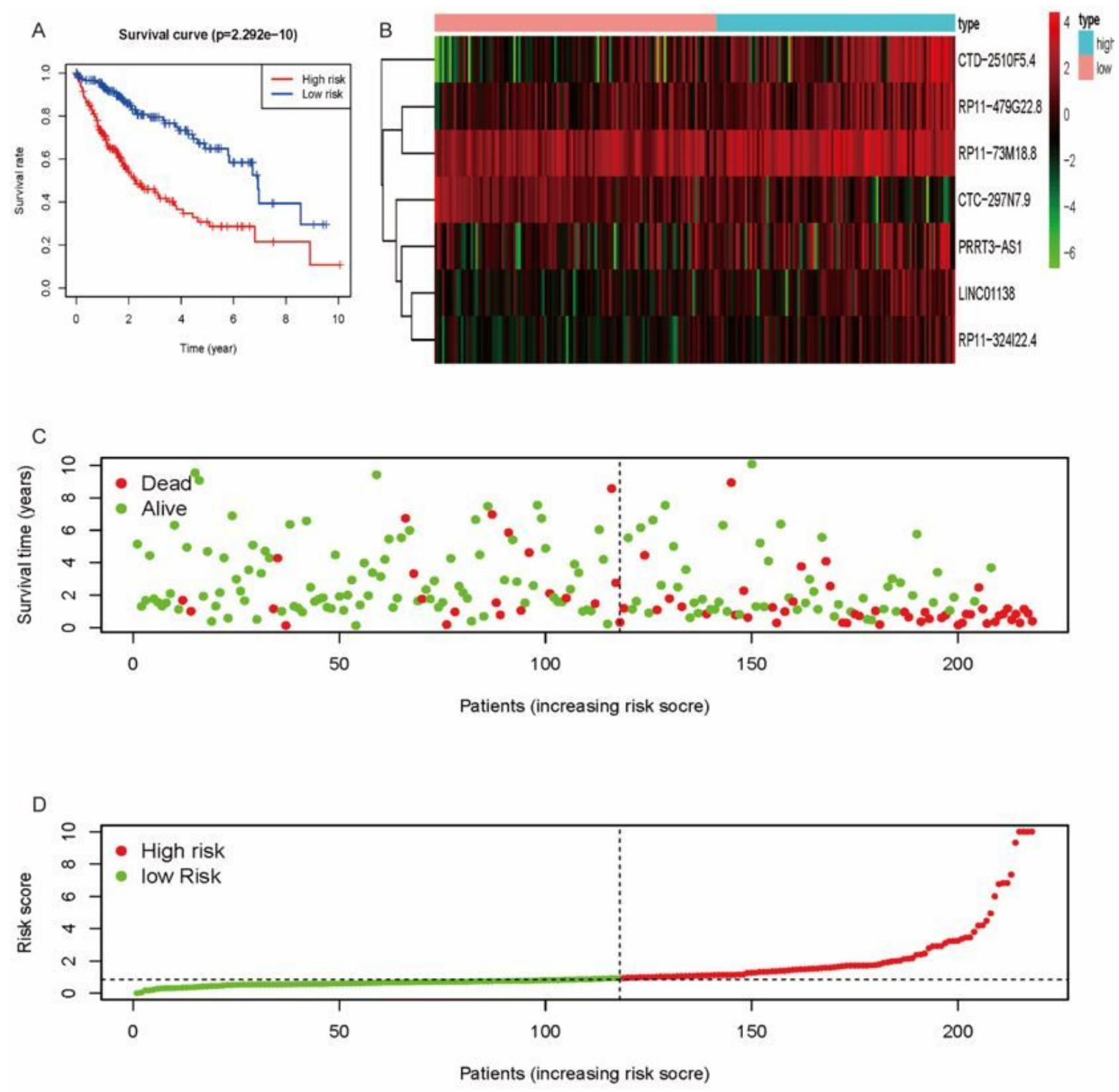

\section{Figure 3}

The analysis of autophagy-related IncRNA for patients with hepatocellular carcinoma. (A) The KM survival curve of the 7 autophagyrelated IncRNA. (B) The Heatmap of 7 autophagy-related IncRNAs' expression. (C) The scatterplot of the risk scores and the survival status/survival time. Red represents dead; green represents alive. (D) The ranked risk score of all autophagy-related IncRNAs. Red represents a high risk; green represents a low risk. 
A

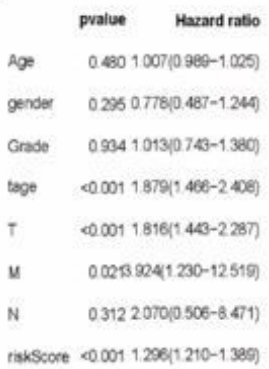

B

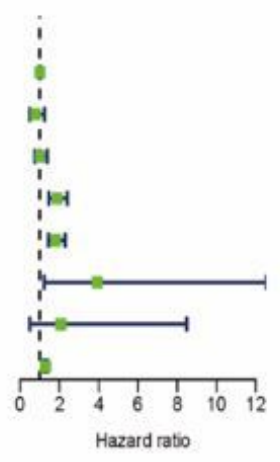

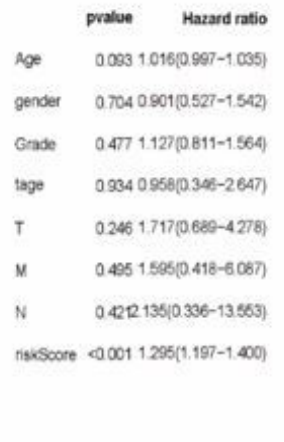
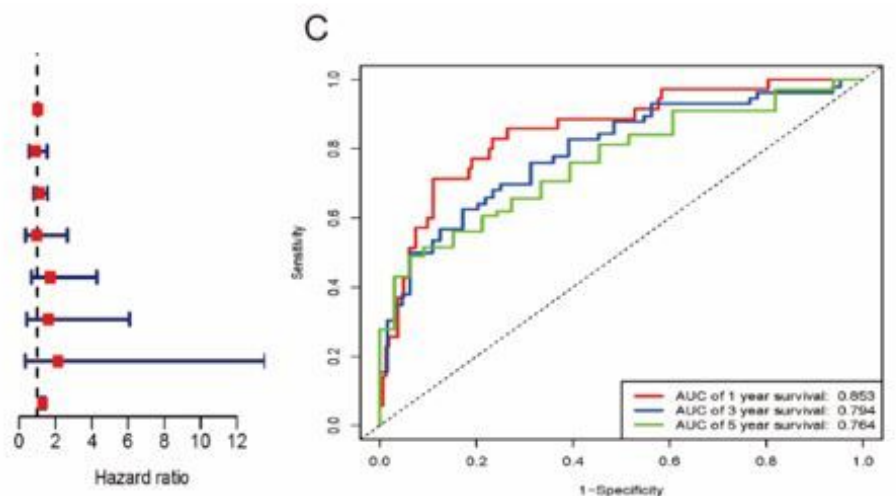

\section{Figure 4}

Clinical Value of the Prognostic Autophagy-Related IncRNA. The forest plots of HCC based on (A) univariate Cox regression analysis and (B) multivariate Cox regression analysis. (C) The area under the ROC (AUC) curve of 1-, 3-, and 5-year.

A

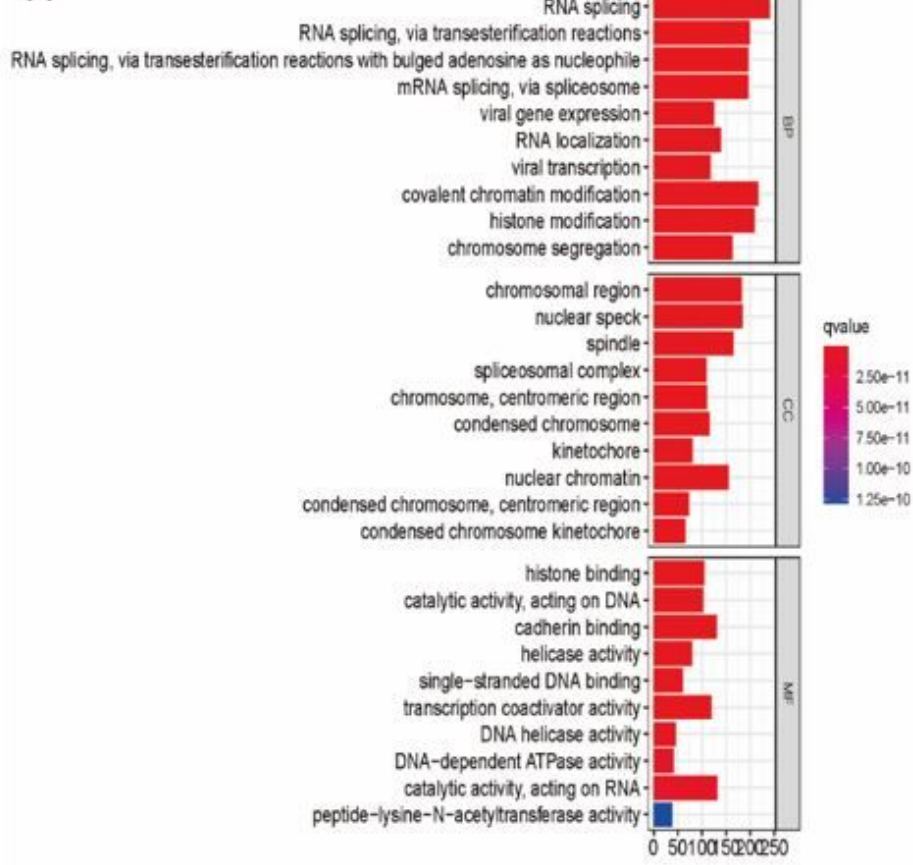

B

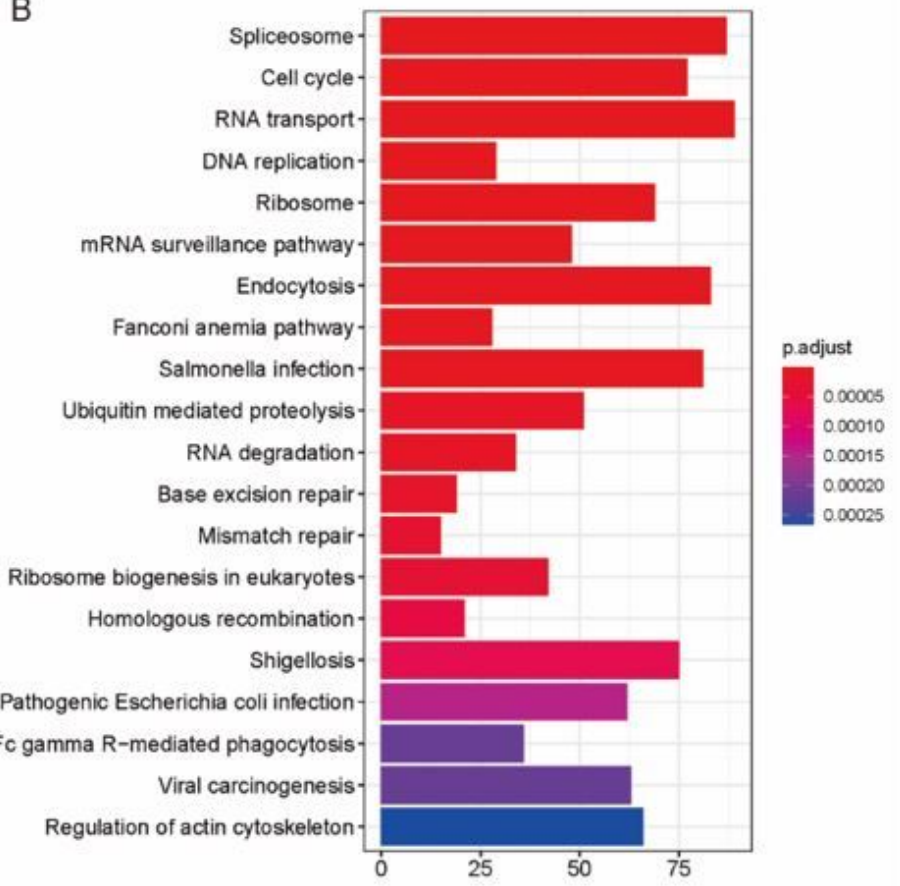

\section{Figure 5}

GO and KEGG pathway enrichment analyses. (A) Bar graph of GO enrichment analysis of the 7 autophagy-related IncRNA. (B) Bar graph of KEGG pathway enrichment analysis of the 7 autophagy-related IncRNAs. GO: Gene Ontology; KEGG: Kyoto Encyclopedia of Genes and Genomes.

\section{Supplementary Files}

This is a list of supplementary files associated with this preprint. Click to download.

- TableS1.ThecoexpressionresoultofautophagyrelatedlncRNAsandautophagygenes.xlsx

- TableS2.The39strongprognosticautophagyrelatedlncRNAsidentifiedbyunivariateCoxregressionanalysisandproductlimitmethod..xlsx

- TableS3.The3580geneshavecoexpressionwith7prognosticautophagyrelatedlncRNAs.xlsx 